\title{
Reversible Switching of Hydrogel-Actuated Nanostructures into Complex Micropatterns
}

\section{Citation}

Sidorenko, A., T. Krupenkin, A. Taylor, P. Fratzl, and J. Aizenberg. 2007. "Reversible Switching of Hydrogel-Actuated Nanostructures into Complex Micropatterns." Science 315 (5811) (January 26): 487-490. doi:10.1126/science.1135516.

\section{Published Version}

doi:10.1126/science.1135516

\section{Permanent link}

http://nrs.harvard.edu/urn-3:HUL.InstRepos:37260716

\section{Terms of Use}

This article was downloaded from Harvard University's DASH repository, and is made available under the terms and conditions applicable to Other Posted Material, as set forth at http:// nrs.harvard.edu/urn-3:HUL.InstRepos:dash.current.terms-of-use\#LAA

\section{Share Your Story}

The Harvard community has made this article openly available.

Please share how this access benefits you. Submit a story.

Accessibility 


\title{
Reversible Switching of Hydrogel-Actuated Nanostructures into
}

\section{Complex Micropatterns}

\author{
Alexander Sidorenko, ${ }^{1}$ Tom Krupenkin, ${ }^{1}$ Ashley Taylor, ${ }^{1}$ Peter Fratzl, ${ }^{2}$ and Joanna Aizenberg ${ }^{1 *}$ \\ ${ }^{1}$ Bell Laboratories/Lucent Technologies, Murray Hill, NJ 07974, USA \\ ${ }^{2}$ Max Planck Institute of Colloids and Interfaces, D-14424 Potsdam, Germany \\ *To whom correspondence should be addressed. Email: jaizenberg@lucent.com
}

\begin{abstract}
:
Responsive behavior, intrinsic to natural systems, is becoming a key requirement for advanced artificial materials and devices, presenting a substantial scientific and engineering challenge. We designed dynamic actuation systems by integrating high-aspect-ratio silicon nanocolumns, either free-standing or substrate-attached, with a hydrogel layer. The nanocolumns were put in motion by the "muscle" of the hydrogel, which swells or contracts depending on the humidity level. This actuation resulted in a fast reversible reorientation of the nanocolumns from tilted to perpendicular to the surface. By further controlling the stress field in the hydrogel, the formation of a variety of elaborate reversibly actuated micropatterns was demonstrated. The mechanics of the actuation process have been assessed. Dynamic control over the movement and orientation of surface nanofeatures at the micron and submicron scales may have exciting applications in actuators, microfluidics, or responsive materials.

Adaptive materials and devices that change properties and function in response to external stimuli are the focus of research in fields ranging from medicine and biology to chemistry, physics, materials science, and engineering. A wide range of artificial responsive materials, mostly involving polymers, has been reported [1-9]. Hydrogels are prominent examples of such materials. Their elastic networks swell in water, allowing one to achieve the desired degrees of polymer hydration and volume changes. A variety of gels that are responsive to humidity, $\mathrm{pH}$, temperature, electric field, light, and ion strength have been designed [1-7] and
\end{abstract}


patterned by means of lithography [8,9]. These responsive gels have been shown to have potential applications in microfluidics, tis-sue engineering, and as shape-memory materials, artificial muscles, valves, and actuators [1-9]. Synthetic routes and fabrication strategies leading to new-generation, dynamically tunable materials are often inspired by biological systems that show a wide range of adaptive responses. Recently, a number of studies have demonstrated that various physicochemical properties of biological materials that are generally vital for survival arise from the presence of highly developed surface nano-roughness and exquisite nano- and microfeatures (10-12). Nano- and microstructures that develop on the surfaces of gecko feet, lotus leaves, and cicada and butterfly wings; for example, provide these organisms with exceptional adhesive, self-cleaning, water-repelling, and photonic properties

Several efforts have been made to artificially produce nanostructured surfaces in polymeric materials that mimic biological structures and functions [13, 14]. The intrinsic flexibility of polymers frequently leads to undesired design outcomes, because the features in soft materials are poorly controlled in general and are often susceptible to irreversible collapse [15]. We reported an alternative approach to the fabrication of nanostructured surfaces, in which regular arrays of well-defined nanostructures with feature sizes of $\sim 100$ to $300 \mathrm{~nm}$ and aspect ratios reaching 100 were formed in silicon by means of the Bosch process [16]. These structures are stable and their geometry is highly controlled. This rigidity, however, makes them unsuitable for use in adaptive materials and devices. Amore promising approach is to create materials based on a hard/soft combination that capitalize on the properties of both elements. Stable superhydrophobic surfaces have been fabricated by using polyelectrolyte multilayers overcoated with silica nanoparticles [17]. Interesting examples of chemically, magnetically, and light-responsive photonic crystals, which were made by dispersing rigid colloidal particles into hydrogels, have 
been reported [18, 19]. Rod-like building blocks consisting of gold and polymer domains have been shown to self-assemble into a variety of superstructures [20].

We propose here to use the combination of soft and hard elements to enable reversible actuation of rigid surface nano- and microstructures that are set in motion by the polymer layer. We integrated a hydrogel (Fig. 1A) with an array of isolated, high-aspect-ratio rigid structures (AIRS) (Fig. 1B) into hydrogel-AIRS assemblies (HAIRS) (Fig. 1, C to E). The AIRS provide rigidity, structure, and precision, whereas the hydrogel provides responsive behavior. We anticipated that the combination of the two would offer substantial dynamic responses based on geometrical rearrangements of the hydrogel-actuated nanostructures

To achieve these designs, the hydrogel was formed in the confinement between AIRS and a secondary substrate (Fig. 1C) [21]. Polyglycidyl methacrylate (PGMA) partially modified with acrylic acid, which forms an anchoring interface that is rich in reactive epoxy groups [22], was chemisorbed on one of the substrates. A layer of polyacrylamide gel (PAAG) grafted to the substrate via PGMA was formed by photo- or thermos-initiated in situ radical copolymerization of acrylamide and N,N'-methylene-bisacrylamide as a cross-linking agent (fig. S1). The AIRS used in our experiments consisted of square arrays of vertically oriented, uniform nanocolumns with diameters $\mathrm{d}=100$ to $300 \mathrm{~nm}$, heights $\mathrm{h}=5$ to $8 \mu \mathrm{m}$, aspect ratios $\mathrm{h} / \mathrm{d}=15$ to 80 , and periodicities $\mathrm{p}=2$ to $4 \mu \mathrm{m}$ (Fig. 1B).

We developed two hybrid architectures using either free-standing (HAIRS-1) or attached (HAIRS-2) nanocolumns. The key element of the HAIRS-1 design is the use of hydrogelembedded silicon nanocolumns that detach from the original AIRS silicon wafer (Fig. 1D). To achieve the HAIRS-1 structure (fig. S2), the anchoring layer of chemisorbed PGMA and acrylic acid was applied to the second surface, whereas the AIRS remained unmodified. The prepolym- 
erizate solution was introduced between AIRS and the modified confining surface. After polymerization, the two surfaces were separated by applying shear stress to the "sandwich" that resulted in the complete breaking of the columns at the base. The detached columns remained embedded in the hydrogel film grafted via PGMA to the confining surface. This procedure was followed by rinsing and drying of the hydrogel to remove unreacted monomers and the solvent, causing the contraction of the film and the associated slanting of the unattached nanocolumns (Fig. 2, A and B). The surface revealed domains with non-uniform tilt directions, conceivably induced by inhomogeneity of the hydrogel film and/or local-surface defects. The tilt angle $\alpha$ of the columns observed in several experiments was 60 to 75 degrees, and the exposed length $l$ was 1.5 to $2.5 \mu \mathrm{m}$. Exposure to humidity led to swelling of the hydrogel and relaxation of the drying stresses. As a result, the tilted columns moved to their original orientation perpendicular to the surface (Fig. 2C). Upon drying, the columns returned to their tilted state. By adjusting the humidity level using the humidity chamber (and thus regulating the degree of hydration and the related volume change of the hydrogel), we can control the tilt angle of the nanocolumns.

To measure the actuation time, the process was recorded with a high-speed video camera [21]. When a water droplet is placed on a dry sample, the switching to the perpendicular orientation is extremely fast ( $\sim 60 \mathrm{~ms})$. Movie S1depicts the process slowed down by about a factor of 7. The reverse transformation to the tilted state caused by unassisted drying of the sample takes $\sim 4$ s. Movie S2 depicts the re-verse process slowed down by a factor of 2.The latter reorientation can be accelerated, if needed, by using airflow in the system and/or by increasing the temperature. The actuation cycle was repeated on the same sample more than 20 times immediately after the preparation and more than 25 times 6 months later. No deterioration or change in the response time was observed, demonstrating a remarkable stability and robustness 
of the designed actuation system. When the anchoring layer of chemisorbed PGMA and acrylic acid isapplied to the AIRS substrate and the confining surface is unmodified, the hydrogel film is grafted to the nanostructured surface (fig. S2). In this HAIRS-2 design (Fig. 1E), the hydrogelembedded nanocolumns remain attached to the original substrate and bend upon the drying of the polymer film (Fig. 3A). The actuation cycle is similar to that described for HAIRS-1: Exposure to humidity causes the hydrogel to swell and relax, thus returning the nanocolumns to their perpendicular orientation. To visualize the reorientation process and the dependence of the tilt angle on the degree of hydration and/or humidity, we placed a droplet of water on the HAIRS-2 surface and took an optical micrograph of the drying edge (Fig. 3B).The water content and the swelling of the hydrogel gradually decrease across this region, inducing a progressive increase in the tilt angles of the nanocolumns.

We further capitalized on these new actuation systems by expanding their capabilities to form reversibly actuated micropatterns with complex geometries. Because the tilt direction of the nanocolumns in the HAIRS-1 design depends on the local defects on the confining surface, we anticipated that this orientation could be controlled by deliberately introducing a topographic pattern onto this surface. Indeed, a highly uniform tilt direction of the nanocolumns was achieved by using a confining surface that was patterned with lines (Fig. 4A). A variety of more complex patterns can be achieved by using an underlying substrate of corresponding topography. One such example is given in Fig. 4B, where a honeycomb-like pattern on the confining surface controls the formation of a complex structure in which arrays of radially oriented columns form "microflowers" that follow the geometry of the templating surface. An array of "microtraps" in which every group of four attached nanocolumns of the HAIRS-2 design is held together by a hydrogel, is depicted in Fig.4C. Such complex patterned movements would be impossible in the 
reported artificial muscle systems, in which the polymers are actuated by an electric or magnetic field. Even more important, these patterns are reversible, in contrast to the cases of structural rearrangement and assembly reported in [20, 23]. Structures shown in Fig. 4, B and C, open and close depending on the humidity level, as illustrated in Fig. 4, D and E. Their movements resemble the responsive behavior of carnivorous plants or pedicellaria (multijawed microforcepslike structures found on echinoderms), whose movement between the open and closed states functions in feeding and defense and as an antifouling mechanism, pre-venting the settlement and growth of microorganisms [24]. These biological structures essentially exist as arrays of environmentally responsive actuators, as do our artificial dynamic patterns. The potential of these unique structures for various applications is now being explored [25]. From a mechanical viewpoint, there is a major difference between the HAIRS-1 and HAIRS-2 systems in the way that the nanocolumns are deformed. In the case of the HAIRS-1 system, the columns correspond essentially to stiff elements loaded under compression, whereas the shrinking hydrogel exerts tensile forces. This type of design was recognized long ago for its enormous potential in architectural construction by Buckminster-Fuller, who coined the term "tensegrity” [26], and the concept was later proposed as a mechanical model for the cell cytoskeleton [27]. Such tensegrity structures consisting of stiff rods connected by soft springs under tension are currently being investigated in the context of mechanics for their inherent shape memory and related potential as actuators [28].The HAIRS-1 system is a realization of tensegrity at the microscale, where the nanocolumns redirect the tensile forces from the gel (which a priori would just lead to a reduction of the gel-layer thickness) into a lateral actuation (Fig. 1D).The movement $X$ is controlled by the volume change of the gel and the length of the nanocolumns. If $v_{w}$ and $v_{d}$ represent the volume of the gel in the wet and the dry state, respectively, then 
$\cos \alpha=v_{d} / v_{w}$ and $\sin \alpha=x / h$,

so that

$$
x=h\left(1-\left(v_{d} / v_{w}\right)^{2}\right)^{1 / 2} \text {. }
$$

With the dimensions typically used in this study, the lateral actuation is on the order of $X$ $=4$ to $8 \mu \mathrm{m}$. A more complex topography of the confining surface, alternative (for example, nonquadratic) arrangements of the array of nanocolumns, the use of nanostructures of a different shape (such as blades), or a combination thereof allow for a variety of reversible patterned movements at the micrometer scale (Fig. 4, A and B), which might be technically explored, such as in the context of microfluidic or photonics [25].

HAIRS-2 systems go beyond the concept of tensegrity, in the sense that the beams do not stay straight and carry compressive loads, but instead they bend (Fig. 1E). Typically, the stiffness of thin beams is much less in bending than in compression (assuming that no buckling occurs). As a consequence, a large fraction of the deformation energy is stored in the bent columns, which tend to go back to their straight shape when the tensile stresses from the gel are released upon rewetting. The amount by which each column bends is a measure of the force exerted by the gel. This is in contrast to the HAIRS-1 system, where the force developed by the gel has no direct influence on the structure after drying. Using simple beam theory, we estimated the force $f$ at which the bending of the silicon nanocolumns in the HAIRS-2 design occurs (figs. S4 and S5). This force depends very strongly on the aspect ratio. For an $8 \mu \mathrm{m}$ high column with an aspect ratio of either $80,40,20$, or 10 , the bending occurs at $\sim 50 \mathrm{nN}, \sim 500 \mathrm{nN}, \sim 8 \mu \mathrm{N}$, or $100 \mu \mathrm{N}$, respectively. Moreover, the strain at the base cannot exceed the fracture strain of silicon. The consequence is that columns with a small aspect ratio can only undergo relatively small bending 
and then must break, whereas columns with an aspect ratio of 80 can fully bend to touch the surface without breaking. An aspect ratio of at least 20 is needed for sufficient bending of each beam to touch its neighbors to obtain the structure shown in Fig. 4, C and D. This mechanical assessment makes it possible to design a hydrogel/nanocolumns combination that enables a desired degree of directed actuation. We have developed hybrid architectures in which arrays of high-aspect-ratio silicon nano-columns, either attached or free-standing, are embedded into a hydrogel film and are actuated into highly controlled, complex microstructures upon contraction and/or swelling of the polymer. The actuation is fast, reversible, reproducible, and robust. We believe that these architectures may lead to a variety of applications, including actuators, controlled reversible pattern formation, microfluidics, reversible switching of the wetting behavior, tunable photonic structures, artificial muscles, and release systems [25].

\section{References}

1. S. Minko, Responsive Polymer Materials: Design and Applications (Blackwell Publishing, Ames, IA, 2006) and references therein.

2. Y. Osada, H. Okuzaki, H. Hori, Nature 355, 242 (1992).

3. Y. Osada, A. Matsuda, Nature 376, 219 (1995).

4. D. J. Beebe et al., Nature 404, 588 (2000).

5. T. P. Russell, Science 297, 964 (2002).

6. J. Lahann et al., Science 299, 371 (2003).

7. A. Lendlein, H. Y. Jiang, O. Junger, R. Langer, Nature 434, 879 (2005).

8. F. Chiellini et al., Macromol. Rapid Commun. 22, 1284 (2001).

9. M. Mayer, J. Yang, I. Gitlin, D. H. Gracias, G. M. Whitesides, Proteomics 4, 2366 (2004).

10. T. Sun, L. Feng, X. Gao, L. Jiang, Acc. Chem. Res. 38, 644 (2005) and references therein

11. K. Autum et al., Proc. Natl. Acad. Sci. U.S.A. 99, 12252 (2002) and references therein.

12. P. Vukusic, J. R. Sambles, Nature 424, 852 (2003) and references therein.

13. A. K. Geim et al., Nat. Materials 2, 461 (2003).

14. S. B. Lee, R. Koepsel, D. B. Stolz, H. E. Warriner,A. J. Russell, J. Am. Chem. Soc. 126, 13400 (2004).

15. E. Delamarche, H. Schmid, B. Michel, H. Biebuyck, Adv. Mater. 9, 741 (1997). 
16. . N. Krupenkin, J. A. Taylor, T. M. Schneider, S. Yang, Langmuir 20, 3824 (2004)

17. L. Zhai, F. C. Cebeci, R. E. Cohen, M. F. Rubner, Nanoletters 4, 1349 (2004).

18. A. Asher, in Nanoparticles: Building Blocks for Nanotechnology, V. M. Rotello, Ed. (Kluwer, New York,2004), pp. 145-172.

19. M. K. Maurer, I. K. Lednev, S. A. Asher, Adv. Funct. Mater. 15, 1401 (2005).

20. S. Park, J.-H. Lim, S.-W. Chung, C. A. Mirkin, Science 303, 348 (2004).

21. Materials and methods are available as supportingmaterial on Science Online.

22. K. S. Iyer, B. Zdyrko, H. Malz, J. Pionteck, I. Luzinov, Macromolecules 36, 6519 (2003).

23. B. D. Gates et al., Angew. Chem. Int. Ed. 43, 2780 (2004).

24. E. E. Ruppert, R. S. Fox, R. B. Barnes, Invertebrate Zoology (Brooks Cole Thomson, Belmont, CA, ed. 7, 2004).

25. In particular, our preliminary data show that thesestructures (i) direct the flow and particle transport(analogous to the function of cilia in organisms) and thushave potential in microfluidics, (ii) reversibly changetheir photonic properties and thus can be considered fortunable photonics, (iii) reversibly switch their wettingbehavior from superhydrophobic in the humidenvironment to hydrophilic in the dry environment, and(iv) trap and release particles in microflowers.

26. R. Buckminster-Fuller, Portfolio Art News Annu. 4, 112(1961). Also available at www.rwgrayprojects.com/rbfnotes/fpapers/tensegrity/tenseg01.html.

27. D. E. Ingber, Annu. Rev. Physiol. 59, 575 (1997).

28. M. Defossez, Mech. Res. Comm. 30, 311 (2003).

29. We thank P. Kolodner, J. Weaver, I. Luzinov, and I. Sokolov for fruitful discussions. We thank R. Smith and N. Lippa for technical assistance. This work wassupported in part by the Office of Naval Research, award N00014-05-1-0909.

Figure captions

Figure 1. (A) Schematic presentation of the structure and composition of the PAAG film grafted to the $\mathrm{Si} / \mathrm{SiO}_{2}$ substrate via the PGMA anchoring layer. (B) Scanning electron micrograph (SEM) of a sample AIRS structure composed of an array of silicon nanocolumns. An inset on the right reveals a banding pattern (scalloping) that is characteristic of the Bosch fabrication process. The band width is 200 nm.(C) Hydrogel synthesis in the confinement of the AIRS and a secondary substrate (a glass slide or a silicon wafer, either flat or topographically patterned) separated by a spacer of the desired thickness that regulates the size of the polymer film. (D) Hybrid HAIRS-1 design. The nanocolumns, embedded into the hydrogel layer grafted to the PGMA-modified 
confining surface, break from the original wafer upon the separation of the confining substrates and become fully transferred onto the secondary surface. The surface topography changes from an array of highly tilted to vertically oriented nanocolumns. (E) Hybrid HAIRS-2 design. The hydrogel film is attached directly to the AIRS silicon wafer. Nanocolumns remain attached to the surface and bend upon drying.

Figure 2. Microscopy study of the HAIRS-1 design.(A) SEM image of a dry sample viewed perpendicular to the surface reveals tilted columns organized in domains with different tilt directions. The top inset shows a zoom-in view perpendicular to the surface. The number of 200 $\mathrm{nm}$ bands $n$ on the emerging portion of the nanocolumns and the length of the column projections $a$ were monitored to determine the length $l$ of the exposed nanocolumns ( $l=200 n$, measured in $\mathrm{nm}$ ) and their tilt angle ( $\sin \alpha=a / l$ ). The SEM of the cross section (bottom inset) clearly shows the tilted nanocolumns partially embedded into the hydrogel layer. Scale bars in insets, $2 \mu \mathrm{m}$. (B and C) Optical micrographs, imaging the same regionof the HAIRS-1 system in a dry (B) and a wet (C) state, show the reorientation of the nanocolumns from a tilted to a vertical position upon the expansion of the hydrogel. Also see movies S1 and S2 and Figure S3.

Figure 3. Microscopy study of the HAIRS-2 structure. (A) Low-magnification SEM (left) and high-magnification SEM (right) show the hydrogel layerthat forms characteristic onion-like or conical features at the bottom of the nanocolumns. (B) Optical micrograph of the drying edge of the HAIRS-2 structure taken perpendicular to the surface. The clarification of the actuation mechanism is shown schematically below the micrograph. A dashed line in the schematic corresponds to the focal plane in the image. The degree of hydration or swelling of the polymer 
layer decreases gradually across the sample from left to right. Correspondingly, the nanocolumns gradually change their orientation from perpendicular to tilted. The whole range of the orientations is depicted, thus providing a still image of the entire reorientation process.

Figure 4. SEM and optical images showing highly controlled pattern formation in the HAIRS designs. All the structures reversibly switch to the original vertical orientation of the nanocolumns upon rewetting. (A) Uniform orientation of the titled nanocolumns in the HAIRS-1 structure templated by the patterned confining surface that is bearing lines. The direction of the lines is indicated by an arrow. (B) Regular array of microflorets in the HAIRS-1 structure templated by the substrate bearing a honeycomb pattern. (C) An example of a complex HAIRS-2 pattern, showing an array of microtraps, in which every group of four attached nanocolumns is held together by the hydrogel. (D and E) Optical micrographs imaging microtraps shown in (C) in a dry (D) and a wet (E) state. The switching of the nanocolumns from bent four-fold clusters to a vertical orientation is clear. 
Figures

Figure 1.

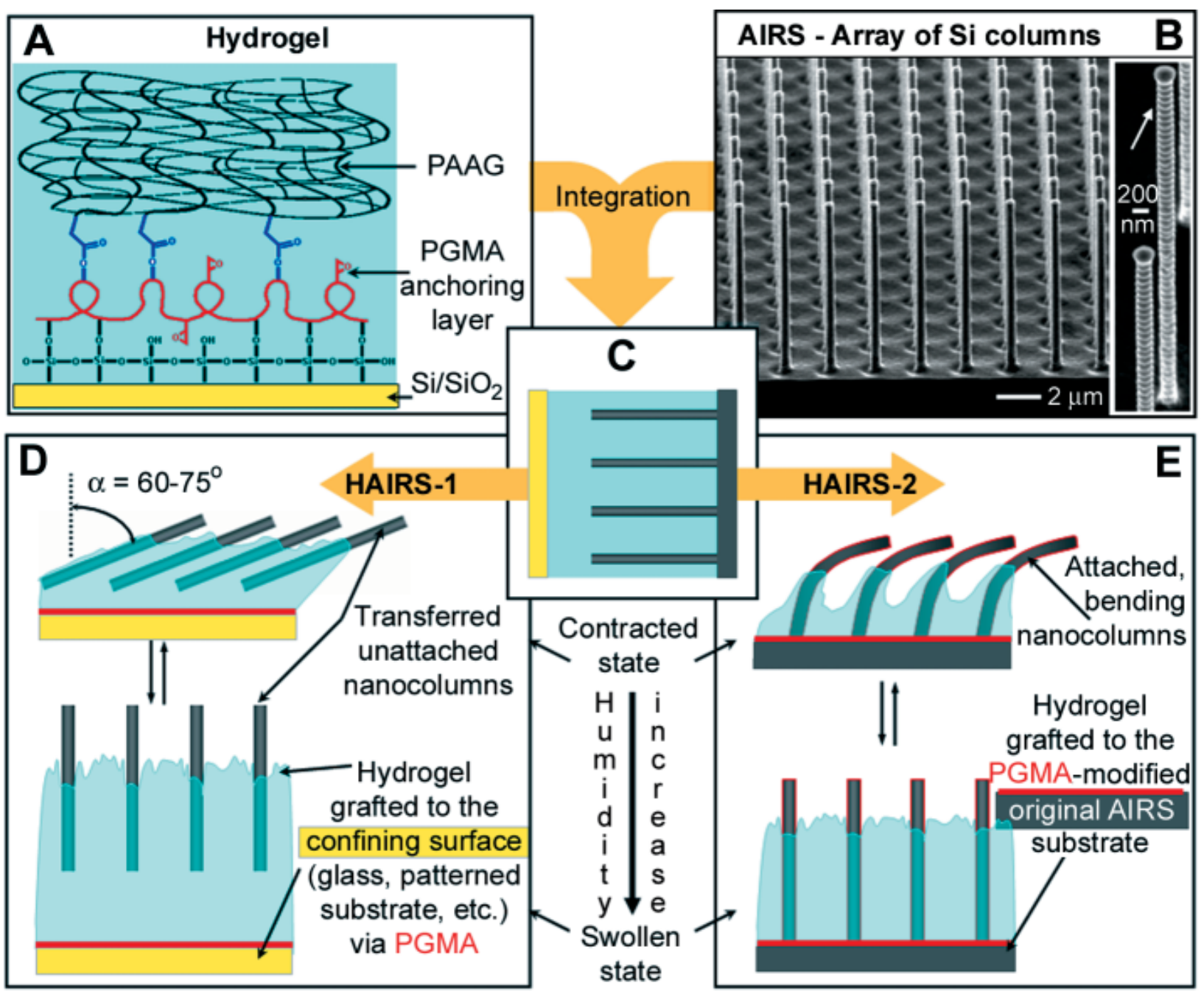


Figure 2.

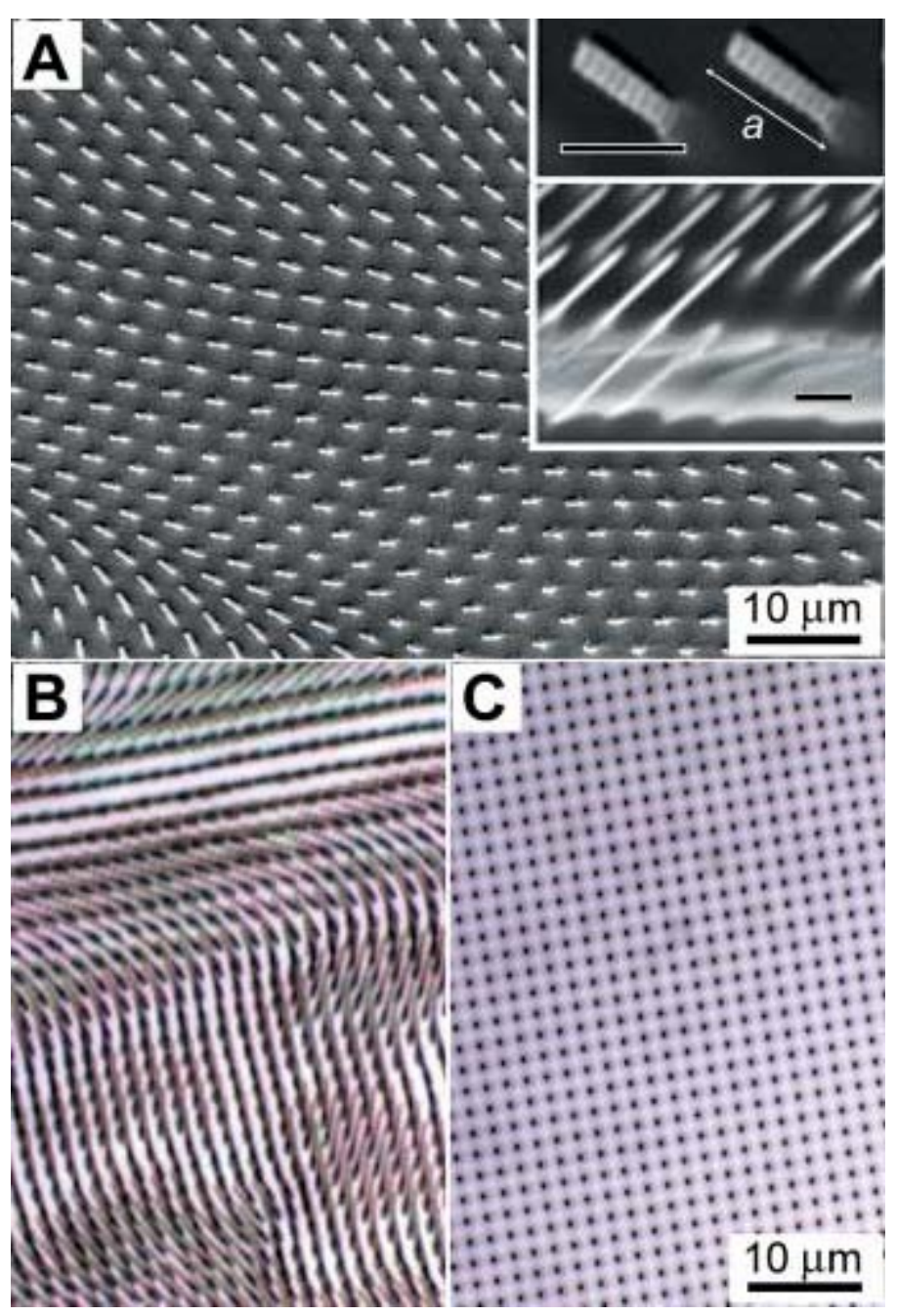


Figure 3.
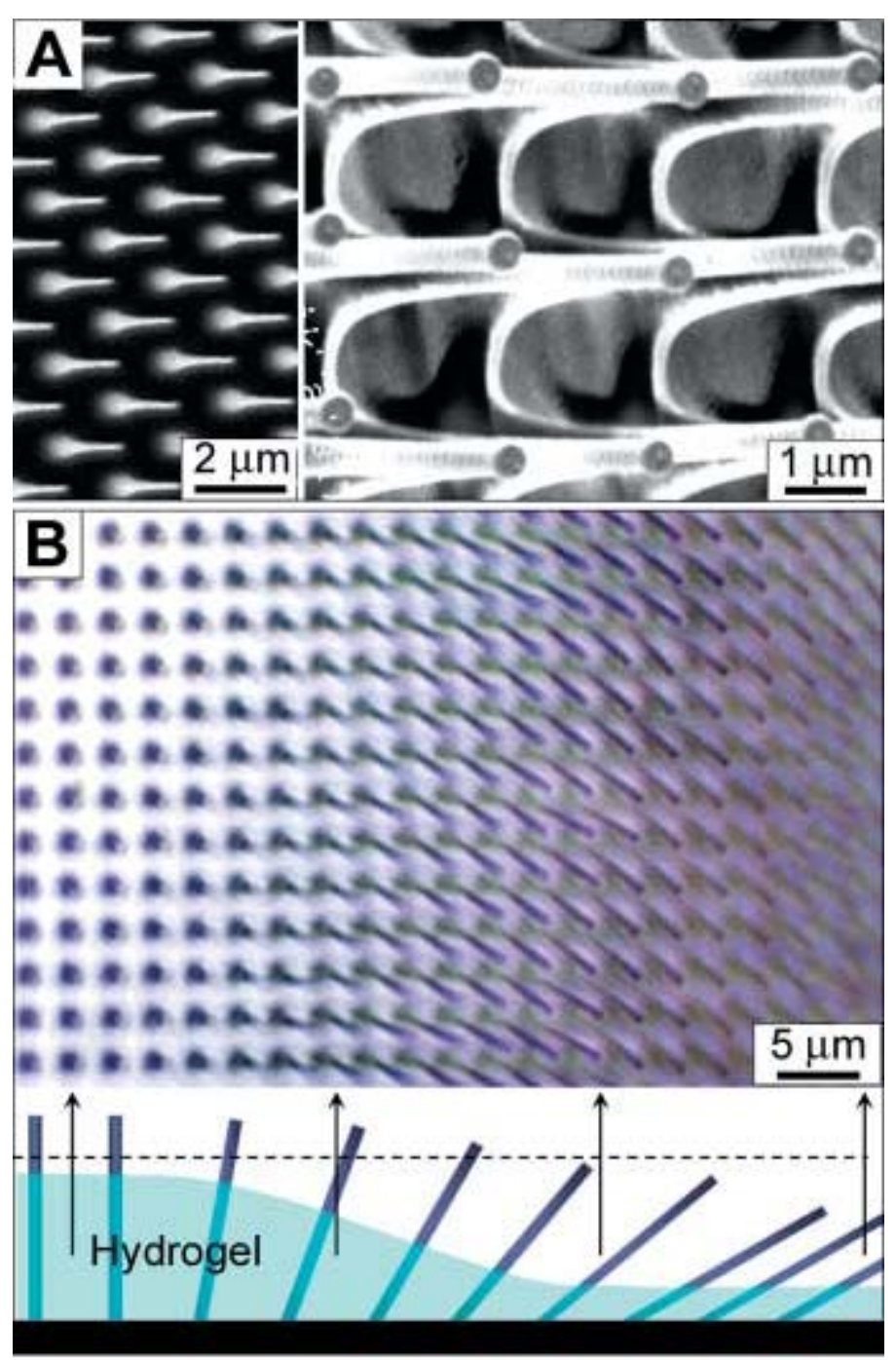
Figure 4.
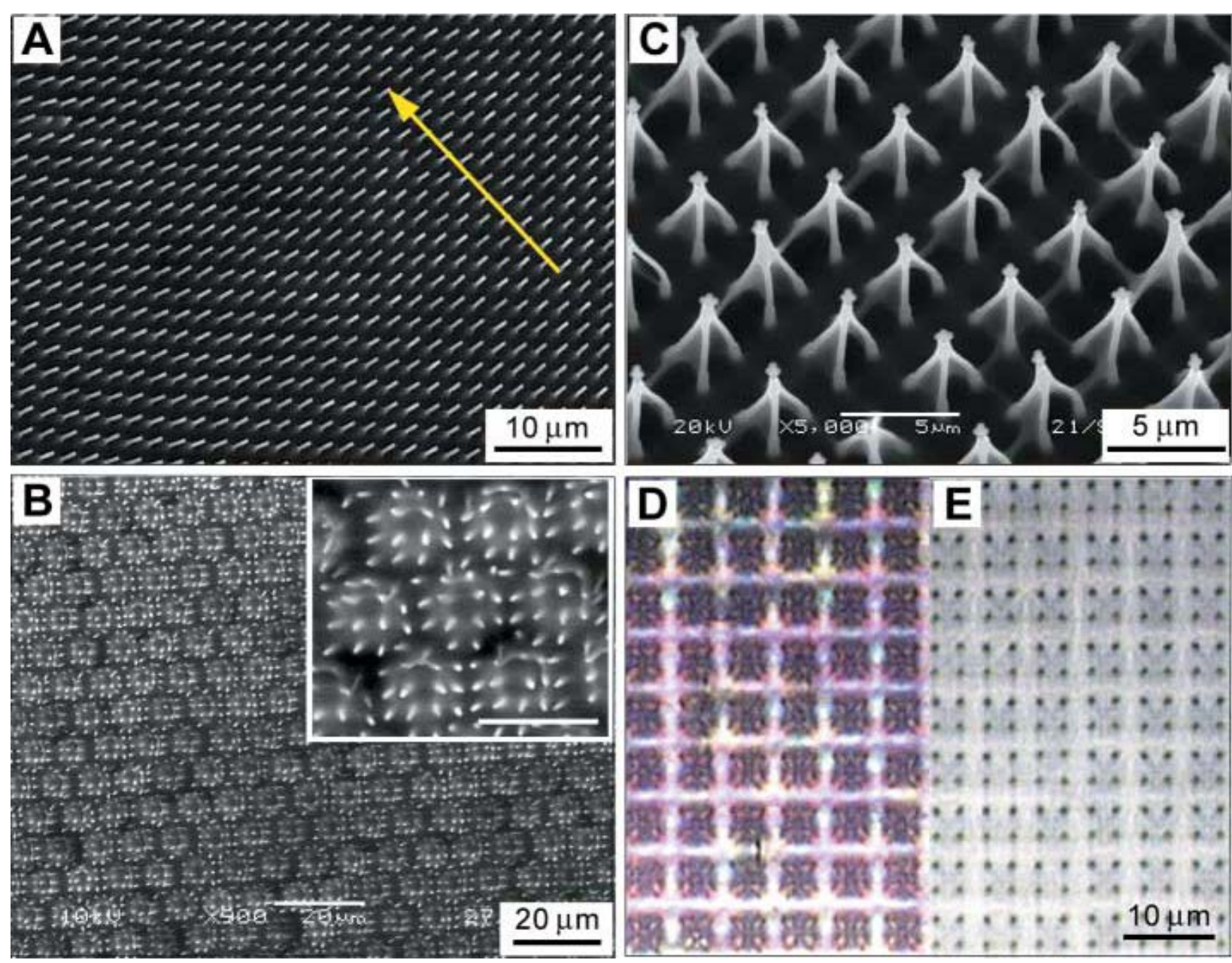